\title{
Deterioro de la función empresarial, debido al síndrome de Dunning y Kruger en el capital humano organizacional del centro de Coahuila
}

\section{Impairment of the business function, due to organizational of Dunning and Kruger syndrome in human capital in the center of the state of Coahuila}

\author{
VÁZQUEZ-LÚNDEZ, Jorge Luis † \\ ID $1^{\text {er }}$ Autor: Jorge Luis, Vázquez-Lúndez / ORC ID: 0000-0002-9256-5759, CVU CONACYT ID: 240054
}

DOI: $10.35429 / J T M S .2019 .16 .5 .36 .44$

Recibido 10 de Junio 2019; Aceptado 30 Septiembre, 2019

\section{Resumen}

El síndrome o efecto de Dunning Kruger (SDK), se caracteriza por la incapacidad de la persona para reconocer su incompetencia e ineptitud, esto ocurre en los ámbitos del quehacer laboral humano. Es una distorsión cognitiva que se refleja al realizar determinada actividad, este tipo de persona cree que tiene mucho conocimiento, incluso más que los expertos; de tal modo que cuanto menos sabe que tiene el síndrome en cuestión, tendrá menos capacidad para reconocer su poco conocimiento y su limitación; en consecuencia, la infundada confianza en sí mismo, le hace creer que es altamente competente. Esta limitante, impide que la persona reconozca su propia incompetencia. El presente estudio, se desarrolla en organizaciones del centro del estado de Coahuila, México; como objetivo, se da a conocer la afectación que (SDK), ocasiona en la consciencia laboral y en la efectividad y mostrar que su efecto aparece en la mayoría de las organizaciones, provocando inhabilidad real y bajo rendimiento en los resultados del trabajo en todos los niveles, utilizando una metodología de investigación de tipo mixto; la contribución del estudio es dar a conocer y proporcionar información confiable sobre dicho síndrome, útil a la organización y aportar en favor de sus objetivos.

\author{
Síndrome de Dunning Kruger, Distorsión \\ cognitiva, Consciencia laboral
}

\begin{abstract}
Syndrome or effect of Dunning Kruger (SDK), is characterized by the inability of the person to recognize his incompetence and ineptitude, this happens in areas of human laborer endeavor. It is a cognitive distortion that is reflected to perform certain activities, such a person believed to have much knowledge, even more than experts; in such a way that the less known to have the syndrome in question, you will have less ability to recognize your little knowledge and its limitation; as a result, unfounded confidence in itself, makes you believe that it is highly competent. This constraint, prevents the person to recognize their own incompetence. This study, takes place in the center of the State of Coahuila, Mexico, as an objective, is given to know that (SDK), It causes consciousness and effectiveness and show showing its effect in most organizations, causing inability to actual and underperformance in the results of the work at all levels, using a mixed-type research methodology; the contribution of the study would be to publicize and provide information about this syndrome, in the Organization and provide for their objectives.
\end{abstract}

Syndrome of Dunning Kruger, Cognitive distortion, Labor consciousness

$\dagger$ Investigador contribuyendo como primer autor. 


\section{Introducción}

Existen personas que muy poco valoran sus capacidades y competencias reales, por lo cual es posible decir que esto se produce debido al efecto de que se tiene un falso conocimiento de autovaloración lo más completo posible, por el cual se tiende a pensar que "todo el mundo lo hace igual", asumiendo así que sus habilidades se encuentran dentro del promedio. Sin embargo, en realidad las habilidades de estas personas son claramente inferiores y que su estimación de sus propias capacidades puede estar errada en uno u otro sentido: bien porque se sobrestima o bien porque valoran muy bajo dichas capacidades; este efecto es señalado por Dunning y Kruger, en relación con que, las personas incompetentes "no solo llegan a conclusiones equivocadas que los llevan a tomar malas decisiones, sino que su incompetencia no les permite tomar conciencia de ello.

\section{Justificación y enfoque}

Ocasionalmente la responsabilidad sobre los fracasos que se experimentan a lo largo de la vida no son realmente debidos al resto de la gente ni a la mala suerte, sino a la persona misma y a sus malas decisiones. Para ello sería conveniente tener la posibilidad de realizar ejercicios de autoevaluación para encontrar este tipo de obstáculos en alguna actividad que se pretenda realizar.

Sobre el conocimiento y dominio del conocimiento laboral, nadie es absolutamente experto en todas las disciplinas de conocimiento relativas a la actividad laboral que se desarrolla, en todos los campos existen carencias y se ignoran muchos aspectos sobre ciertos temas. Cada persona tiene potencial de mejora en cualquier punto de su etapa vital, la verdadera falla es pretender saberlo todo.

Las afectaciones que el efecto señalado por Dunning y Kruger, según el cual personas con escaso nivel intelectual y cultural tienden sistemáticamente a pensar que saben más de lo que realmente saben, considerándose más inteligentes de lo que son y a sobrestimar por definición las capacidades y conocimientos de los demás, ocurre cuando un elemento incómodo habitual en el ambiente laboral presenta un peligro real, cuando asciende a su máximo grado de incompetencia jerárquica.

\section{Objetivo General}

Ofrecer un marco referencial y objetivamente informativo, desde la experiencia real de las Organizaciones, Con el estudio de las principales variables que generan la aparición de conductas debidas al efecto Dunning- Kruger, llamado también síndrome de kruger, que se vive hoy en día, afectando a las organizaciones.

\section{Tipo de investigación}

Como investigación descriptiva el presente artículo pretende identificar las relaciones que existen entre los hechos para lograr una comprensión del efecto Dunning-Kruger y como investigación explicativa realiza la descripción de conceptos o fenómenos o de dicho efecto; efectuando aplicaciones de tipo mixto, combinando aspectos de teoría con el ejercicio de comprobación estadística.

\section{Sustento teórico}

El efecto Dunning-Kruger es un fenómeno sicológico según el cual las personas con escaso conocimiento tienden sistemáticamente a pensar que saben mucho más de lo que saben y a considerarse más inteligentes que otras personas más preparadas, debido a que su propia incompetencia les dificulta reconocer sus errores y evaluar la competencia de los demás. En otras palabras, la gente ignorante o no calificada tienden a sobreestimar su nivel de competencia y experiencia, mientras que los que son verdaderamente expertos muchas veces subestiman su verdadero nivel de experiencia.

El fenómeno fue demostrado en una serie de experimentos realizados por Justin Kruger y David Dunning, a través de investigar cierto número de estudios que tendían a sugerir que en diversas habilidades como la comprensión lectora, conducción de vehículos de motor, y juegos como el ajedrez o el tenis, "la ignorancia frecuentemente proporciona más confianza o seguridad que el conocimiento".

El efecto demuestra que : 1. Los individuos incompetentes tienden a sobreestimar su propia habilidad. 2. Los individuos incompetentes son incapaces de reconocer la habilidad de otros. 3. Los individuos incompetentes son incapaces de reconocer su extrema insuficiencia. 
4. Si pueden ser entrenados para mejorar sustancialmente su propio nivel de habilidad, estos individuos pueden reconocer y aceptar su falta de habilidades previa. Desde su propuesta y nombramiento, se ha convertido en un efecto conocido en la psicología cognitiva, y la gente se ha vuelto más consciente de su existencia en los últimos años, debido a los no expertos que tratan de callar a la gente con experiencia, o demagogos que utilizan la etiqueta de "elitismo" para impulsar sus políticas, ya que ridiculizan a los expertos que los desafían. Mientras tanto, la gente con conocimiento real tiende a subestimar su propia competencia. (Figuerola, 2019)

Este curioso síndrome o efecto, como algunos lo llaman, fue establecido por los dos psicólogos que le dan nombre, Justin Kruger y David Dunning, ambos de la Universidad de Cornell en Nueva York.

Debo decir que es un síndrome que me fascina, y que desde que sé de su existencia, lo ves por doquier si te fijas bien. Lo curioso también de este síndrome, es que, en algunas ocasiones, incluso siendo conscientes de ello todos podemos "pecar" de tenerlo en algún momento.

Dicho fenómeno se basa principalmente en la escasa habilidad de un individuo para reconocer su propia ineptitud, sobrevalorando su incompetencia y su escasa habilidad o conocimientos.

\section{Dunning-Kruger Effect}

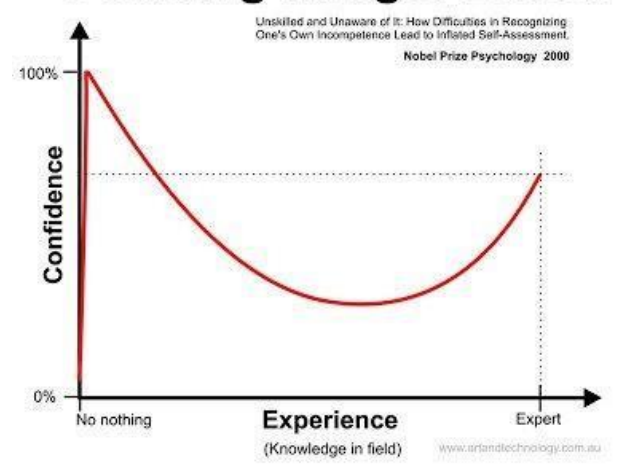

Se da sobre todo en individuos de escasa inteligencia $y$ de escasa cultura, que paradójicamente creen saberlo todo, tener siempre la razón, no ver "más allá de su nariz" y no tener la capacidad de valorar positivamente la capacidad o logros de otras personas con mayor habilidad que ellos.

\section{De acuerdo a Charles Darwin:}

La ignorancia genera más confianza que el conocimiento.

Según los estudios realizados sobre diversos sujetos se sacan, a priori, cuatro conclusiones:

Los individuos incompetentes tienden a sobrestimar su propia habilidad.

Los individuos incompetentes son incapaces de reconocer la habilidad de otros.

Los individuos incompetentes son incapaces de reconocer su extrema insuficiencia.

Si pueden ser entrenados para mejorar sustancialmente su propio nivel e habilidad, estos individuos pueden llegar a reconocer $y$ aceptar su falta de habilidades previa.

Dado que es un síndrome que se da en gran parte de la población, y dado que las personas inteligentes siempre dudan de sí mismas, una persona con inteligencia estará rodeada de personas de este tipo, que minarán aún más su confianza y se sentirá "fuera de lugar".

Conocer de este síndrome puede ayudar a las personas con cultura a darse cuenta de ello e intentar sobrellevarlo; pero cuidado, porque incluso los más inteligentes pueden incurrir en este síndrome en algún momento, ya que puedes ser un experto en un tema o la persona más capaz del mundo en otra habilidad y sin embargo no estar capacitado para darte cuenta de que no eres tan bueno en otros temas.

Hay que tener en cuenta también que antes de valorar negativamente las opiniones, es debido considerar que los sujetos que presentan este fenómeno no están capacitados psicológicamente para ser capaces de darse cuenta de ello.

Bertrand Russel indicó sobre esto, que uno de los dramas de nuestro tiempo está en que aquellos que sienten que tienen la razón, son estúpidos y que la gente con imaginación y que comprende la realidad es la que más duda y más insegura se siente. 
$\mathrm{Y}$ es que, como dice una frase popular, "no hay más ciego que el que no quiere ver" y un claro ejemplo de ello podemos tenerlo en los resultados de los estudios que se hicieron a sujetos en cuestión en diversas ramas teóricoprácticas.

Resulta que, tras los exámenes al respecto, los individuos que más se sobrestimaban sacaron unas notas que los colocaban entre el $12 \%$ de los peores, ellos consideraban que sus notas estarían entre el $62 \%$ mejores.

$\mathrm{Y}$ este efecto se incrementa exponencialmente, cuando ya el individuo en cuestión, aun sabiéndose errado e incluso siendo consciente de su poca capacidad, crea la mayor de las fantasías para hacer creer que tiene razón y que es mejor. Da igual que le pongas un color negro delante, él lo verá blanco e incluso buscará gente para que lo vea blanco como él.

Cualquier cosa antes de aceptar su deficiencia. Vanidad y desconocimiento profundo, unidas en una peligrosa combinación. En cualquier caso, y dado que cualquier persona puede estar en mayor o menor grado padeciendo este síndrome durante algún momento, la humildad puede ayudar a comprenderlo junto con la citada formación.

Incluso estos "ciegos de conocimiento", podrán darse cuenta de ello y se estará en el camino a formar parte de un grupo más avanzado, precisamente que puedan ser capaces de ver las mencionadas limitaciones. (Cabanes, 2017).

En los procesos de aprendizaje en estudios avanzados (de grado o de post-grado) no es solamente necesario que el estudiantado adquiera los conocimientos requeridos, sino que además le deben ser proporcionadas las herramientas necesarias para poder autoevaluarse adecuadamente, capacidad que le será necesaria para su adaptabilidad en un contexto de aprendizaje permanente. El conocido como efecto Dunning-Kruger (Kruger y Dunning, 1999; Ehrlinger y Dunning, 2003), describe la percepción exageradamente alta de las propias capacidades en las fases iniciales de adquisición de conocimientos en una materia, así como la percepción más baja de lo que correspondería por parte los expertos en ella.
Este efecto tiene implicaciones sobre el proceso educativo, puesto que en el primer caso la capacidad del alumnado de evaluar su propia competencia queda mermada por la falta de herramientas (experiencia o conocimientos) necesarias para ello, mientras que los instructores (como expertos) pueden juzgar las tareas que encomiendan a los estudiantes como más asequibles de lo que realmente son. Por otro lado, en cuanto a la evaluación del conocimiento de otras personas en relación a la percepción del propio conocimiento, algunos experimentos (Ames y Kammrath, 2004) demuestran que una mayor percepción de propia competencia redunda en una más baja evaluación de la competencia de otras personas, explicando este fenómeno a través del narcisismo de los sujetos.

Para investigar la relación entre la propia evaluación, se realizó una experiencia educativa de evaluación entre iguales en el ámbito universitario, en los resultados dónde se pidió al estudiantado que evaluara su propio trabajo y dos trabajos ajenos seleccionados aleatoriamente dentro de la misma clase, tanto mediante el uso de una rúbrica como con una evaluación subjetiva. (Garcia-Almirall, 2015).

El síndrome (efecto) de Dunning Kruger (SDK) atenta contra las premisas básicas de la gerencia de capital humano que dan bienestar y desarrollo a toda organización (Félix, 2007), indicando claramente los siguientes aspectos esenciales:

\section{Productividad. \\ 2. Comunicación. \\ 3. Conducta. \\ 4. Identificación. \\ 5. Motivación.}

Conceptos que serán manejados como variables intervinientes en el presente estudio que se derivan de los conceptos anteriores:

\section{Variables intervinientes}

1. Propensión: alegría acompañada por la idea de alguna cosa que es, por accidente, causa de alegría.

2. Aversión: es una reacción acompañada por la idea de alguna cosa que es, por accidente, causa de rechazo. 
3. Incompetencia: es la ineptitud o torpeza en el desempeño de una actividad, cargo en la realización de un trabajo.

4. Irreconocimiento: acción mental que impide que no se puedan distinguir o Identifica elementos del comportamiento propio o de los demás.

5. Desesperación: es una tristeza crítica nacida de la idea de una cosa futura o pretérita acerca de la cual ha desparecido toda causa de duda.

6. Irresponsabilidad: es una circunstancia de la persona a no poder expresar responsabilidad sobre sobre su actitud o propio comportamiento.

7. Indignación es un sentimiento de odio hacia alguien que ha hecho mal a otro.

8. Sobreestimación: es estimar de más a alguien, por amor, en más de lo justo.

9. Soberbia: es sobreestimarse, por amor de sí, en más de lo justo.

10. Abyección: consiste en sentirse por tristeza en menos de lo justo.

11. Vergüenza: es estado anímico acompañado por la idea de alguna acción que imaginamos que los demás vituperan.

12. Nostalgia: es el deseo o apetito de poseer una cosa, sustentado por el recuerdo de esta cosa y al mismo tiempo reprimido por el recuerdo.

13. Temor: es una respuesta por evitar un mal mayor, del que tenemos miedo, mediante otro menor.

14. Pusilanimidad: Cuando el deseo es reprimido por el temor de un peligro que sus iguales osan arrostrar.

15. Ira: es un arranque anímico que incita al quien lo siente, por odio, a hacer mal a quien se odia.

16. Soberbia: consiste en estimarse de manera extrema, por amor de sí, en más de lo justo.

17. Orgullo: es el exceso de estimación propia de los propios $m$ éritos, por el cual se cree uno superior a los d emás.

18. Audacia: es un deseo por el cual alguien es incitado a hacer algo corriendo un peligro que sus iguales tienen miedo de arrostrar.

19. Odio: es una tristeza acompañada por la idea de una causa externa.
20. Desprecio: es la imaginación de alguna cosa que toca tan poco al alma, que el alma misma, deshecha la presencia de la cosa, cualidad o persona.

Sobre la aplicación de estas variables, se elabora un instrumento a modo de encuesta, a 30 sujetos, en relación con lo observado y/o lo que estos sienten, con respecto a estos comportamientos en su medio de trabajo, los resultados de las pruebas estadísticas se integran y se expresan de manera comprensiva en las conclusines sobre efecto de Dunning Kruger.

\section{Diseño}

El diseño de la investigación que se ha decidido formular una hipótesis, ya que la investigación descriptiva y de tipo mixto, ya que se realizó y aplicó una encuesta.

Ya que el tipo de diseño de investigación que utiliza para su estudio se basará en sus fines y objetivos, que a su vez están contenidos dentro de la hipótesis formulada, además de involucrar aspectos de la propuesta se siguen desde el diseño del estudio, se ha incluido la recopilación y análisis de datos y la determinación del tamaño de la muestra, de tal manera que se podrá considerar positiva la hipótesis de acuerdo con el desarrollo de la investigación propuesta.

\section{El Problema Detectado}

El problema observado es que existe un claro efecto que desencadena incompetencia y baja funcionalidad, en empleados que se desempeñan en organizaciones del centro del estado de Coahuila, debido al síndrome (efecto) de Dunning Kruger (SDK), mostrando un claro deterioro de su eficacia organizacional.

\section{Actividades Generales}

Conocer en que consiste el síndrome.

- Conocer la parte psicológica de cualidades personales y profesionales de los ejecutivos que manifiestan este este síndrome.

- Conocer que otros elementos se combinan con este síndrome.

- Identificar la aparición y conocimiento los daños organizacionales relacionados con la aparición de este síndrome. 


\section{Pasos Metodológicos}

Operacionalización de Variables Aplicación de Instrumentos

\section{Obtención de conclusiones}

1. Identificación de los elementos que detonan la aparición del síndrome en la organización.

\section{Hipótesis formulada}

La hipótesis se expresa de la siguiente forma: $\mathrm{Si}$ existen atributos de soberbia, orgullo e irresponsabilidad en la persona que con su comportamiento indica poseer los componentes básicos del síndrome (efecto) de Dunning Kruger (SDK), en su comportamiento laboral, esto impide que esta persona reconozca su propia limitación profesional (Irreconocimiento); entonces se manifiesta un claro conflicto que desencadena su máximo grado de incompetencia, que deteriora gravemente la eficacia de la organización.

\section{Instrumento de medición}

El instrumento de investigación queda integrado como a continuación se indica:

A. Escala de medición

Se aplica la siguiente escala de medición tipo Likert.

\begin{tabular}{|c|c|c|}
\hline 1 & Ninguna & \\
\hline 2 & Minima & $\Xi$ \\
\hline 3 & Inadvertida & $\stackrel{\pi}{2}$ \\
\hline 4 & Coniderable & $\frac{0}{0}$ \\
\hline 5 & Total & 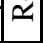 \\
\hline
\end{tabular}

Tabla 1 Escala de medición tipo Likert

\section{Manejo de variables}

Se aplica una encuesta en base a veinte Variables, aplicando una encuesta de veinte preguntas cerradas y precisas a treinta sujetos integrantes de diez organizaciones de la región centro del Estado de Coahuila

\begin{tabular}{|l|l|}
\hline No & CONCEPTO \\
\hline 1 & Propensión \\
\hline 2 & Aversión \\
\hline 3 & Incompetencia \\
\hline 4 & Irreconocimiento \\
\hline 5 & Desesperación \\
\hline 6 & Iresponsabilidad \\
\hline 7 & Indignación \\
\hline 8 & Sobreestimación \\
\hline 9 & Soberbia \\
\hline 10 & Abyección \\
\hline 11 & Vergüenza \\
\hline 12 & Nostalgia \\
\hline 13 & Temor \\
\hline 14 & Pusilanimidad \\
\hline 15 & Ira \\
\hline 16 & Soberbia \\
\hline 17 & Orgullo \\
\hline 18 & Audacia \\
\hline 19 & Odio \\
\hline 20 & Desprecio \\
\hline
\end{tabular}

Tabla 2 Listado de Variables

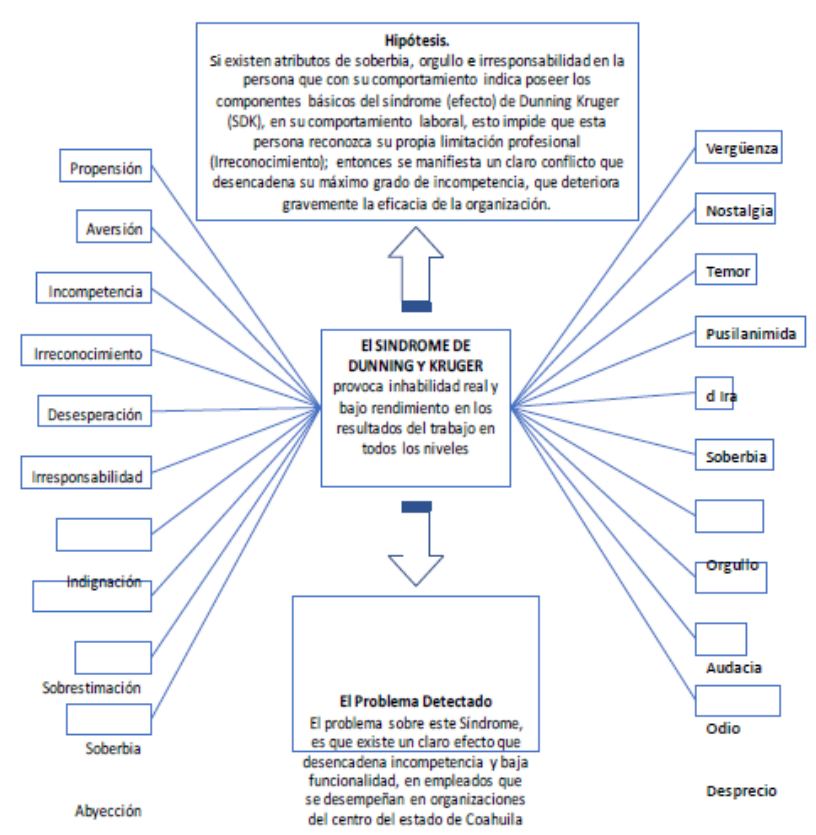

Figura 1 Variables que influyen para la existencia del síndrome de Dunning Kruger (SDK)

Fuente: Elaboración Propia

\section{Población y Muestra}

El desarrollo se inicia con una población de 40 sujetos compuesta por directivos y Empleados de 20 organizaciones productivas de la Región Centro del Estado de Coahuila, indicando una muestra calculada de 30 sujetos, como a continuación se indica: 


\begin{tabular}{|c|c|}
\hline Parametro & Valor \\
\hline $\mathbf{N}$ & 40 \\
\hline $\mathbf{Z}$ & 1.96 \\
\hline $\mathbf{p}$ & $50 \%$ \\
\hline $\mathbf{q}=\mathbf{( 1 - p )}$ & $50 \%$ \\
\hline $\mathbf{e}$ & $5 \%$ \\
\hline \multicolumn{2}{|c|}{ Fórmula de n (Tamaño de la muestra) } \\
\hline Numerador & 38.416 \\
\hline Denominador & 1.0579 \\
\hline $\mathrm{n}=$ & 30 \\
\hline
\end{tabular}

Tabla 3 Calculo para elección de la muestra Fuente: Elaboración Propia

\section{Instrumento de medición}

El instrument de investigación queda integrado como a continuación se indica:

\section{Escala de medición}

Se aplica la siguiente escala de medición tipo Likert

Se aplica la siguiente escala de medición

\section{Análisis de Fiabilidad}

\begin{tabular}{|l|l|}
\hline \multicolumn{2}{|l|}{ Analisis de fiabilidad } \\
\hline $\begin{array}{l}\text { Alfa de } \\
\text { Cronbach }\end{array}$ & $\begin{array}{l}\text { N de } \\
\text { elementos }\end{array}$ \\
\hline 0.724 & 20 \\
\hline
\end{tabular}

Tabla 4 Resultado del cálculo de Alfa de Cronbach Realizado con NCSS:v22

De este análisis se obtiene un Alfa de Cronbach de 0.724, el cual se considera aceptable para la muestra resultante, que expresa el grado de exactitud, consistencia y precisión que posee el instrumento de medición utilizado.

\section{Análisis de frecuencia y porcentaje de los datos}

En relación con 150 opiniones consideradas para las variables primordiales, se obtiene lo siguiente:

\begin{tabular}{|c|c|c|c|}
\hline \multicolumn{4}{|c|}{ FRECUENCIA CONSIDERADA PARA VARIABLES PRIMORDIALES } \\
\hline \multicolumn{2}{|c|}{ escala } & Frecuencia & Porcentaje \\
\hline 1 & NINGUNA & 5 & $3 \%$ \\
\hline 2 & MINIMA & 11 & $7 \%$ \\
\hline 3 & INADVERTIDA & 18 & $12 \%$ \\
\hline 4 & CONSIDERABLE & 53 & $35 \%$ \\
\hline 5 & TOTAL & 63 & $42 \%$ \\
\hline & suma Opiniones & 150 & $100 \%$ \\
\cline { 2 - 4 } & & & \\
\end{tabular}

Tabla 5 Resultado del cálculo de Frecuencia y Porcentaje. (Realizado con NCSS:v22)

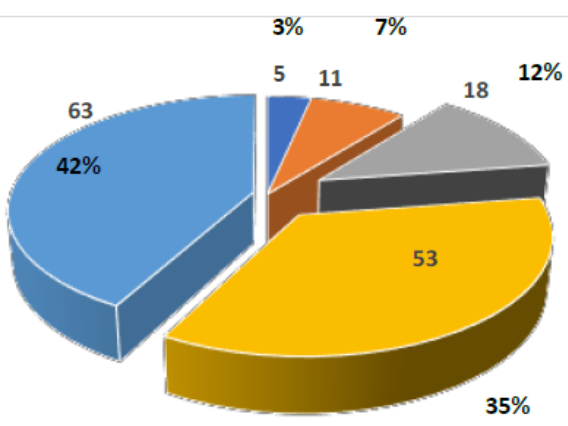

nINGUNA MINIMA INADVERTIDA CONSIDERABLE "TOTAL

Figura 2 Resultado del cálculo de Frecuencia y Porcentaje. (Realizado con NCSS:v22)

\section{Analisis de Normalidad}

Para este análisis se calcula el promedio de cada una de las variables:

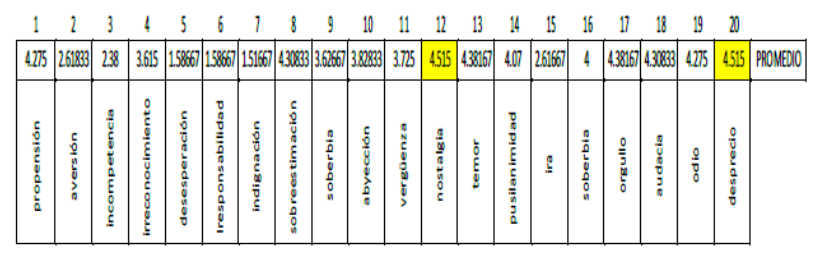
grafica:

Con estos datos se genera la siguiente

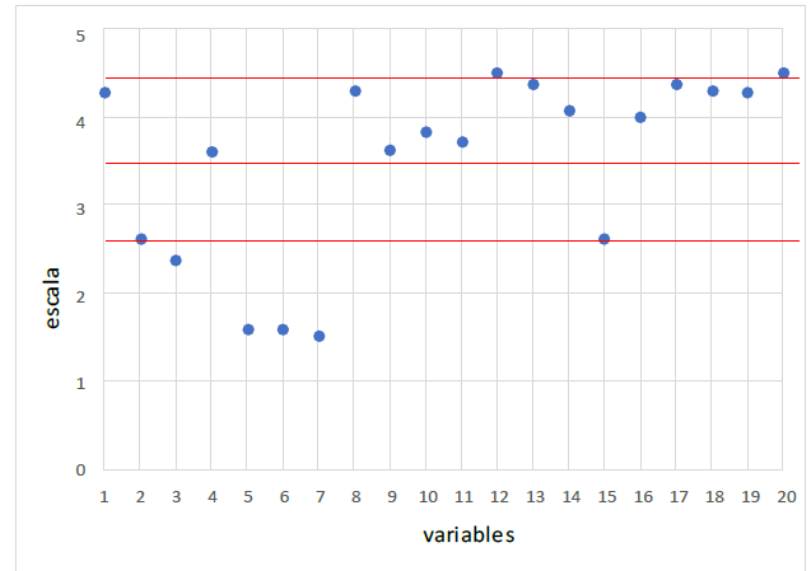

Figura 3 Resultado del cálculo de Normalidad. (Realizado con NCSS:v22)

Como resultado de este análisis, se obtienen las variables 12 y 20 , relativas a nostalgia y desprecio como relevantes, por quedar ubicadas por encima del límite superior de normalidad (4.53). apoyando de esta forma a la hipótesis planteada en ralciión con el conocimiento del síndrome Dunning Kruger (SDK). 


\section{Conclusiones}

Con respecto a lo que indican los autores consultados para este estudio, el síndrome de Dunning Kruger (SDK), atenta contra las premisas básicas de la gerencia de capital humano y al bienestar y desarrollo de toda organización, por otra parte los resultados encontrados mediante los análisis aplicados para esta investigación, mismos que fueron trabajados tomando en cuenta las perspectivas recogidas por las personas que laboran en las empresas participantes y dando sustento a la hipótesis correspondiente relativas a que el síndrome de Dunning Kruger (SDK), cuando existen atributos de soberbia, orgullo e irresponsabilidad en la persona que con su comportamiento indica poseer los componentes básicos de este síndrome, en su comportamiento laboral, esto impide que esta persona reconozca su propia limitación profesional (Irreconocimiento); entonces se manifiesta un claro conflicto que desencadena su máximo grado de incompetencia, que deteriora gravemente la eficacia de la organización, afectando a la mayor parte de los empleados y que es causal de una serie de trastornos en los empleados, que afectan su funcionamiento eficaz.

De acuerdo a los análisis efectuados, se han encontrado congruentes los siguientes supuestos que se basan en que a partir del desarrollo organizacional, la nostalgia, el desprecio, la soberbia y el orgullo desmedidos de los empleados; el síndrome de Dunning Kruger (SDK), afecta negativamente el ambiente organizacional, la productividad de la organización; por otra parte el miedo que forma parte de una cultura organizacional deficiente, puede estar apoyado en este síndrome, generando un impacto negativo importante para la organización.

Por lo que se puede concluir que las opiniones emitidas por los empleados apoyan la existencia y gravedad del síndrome de Dunning Kruger (SDK), aunque en muchos casos existe el desconocimiento de este aspecto en sus organizaciones, que afectan el desarrollo armónico y el bienestar de la misma.

\section{Referencias}

Alvarado, Y., Parra, L., Paz, D., \& Inciarte, D. (2008). Www.produccioncientificaluz.org. Recuperado el 3 de junio de 2015, de www.produccioncientificaluz.org:http://www .produccioncientificaluz.org/index.php/impac to/article/view/6615.

Ahumada S. (2010). Otra vez...el Síndrome de Dunning Kruger (SDK). Gestión del Cambio. 29 de abril de 2010. Accedido el 28 de febrero de 2011 En: http://blog.ahumadaandpartners.com/gestioncambio/otra-vez-el-sindrome-deDunning Kruger (SDK)/.

Burke, W. y Hornstein, H. A. (1972) The Social Techtiology of.... Schmuck, R. y Miles, M. (1971) Organization Development in Schools Carlotto MS, Miralles MDL. (2010). Tradução, adaptação e exploração de propriedades psicométricas da Escala de Adição Trabalho Dutch Work Addiction Scale (DUWAS). Contextos Clínicos 2010; 3: 141-150.

Calot, Gerard. (1988). Curso de Estadística descriptiva. Thompson Paraninfo, S.A. Contreras-Casado D. (2010). Síndrome de Dunning Kruger (SDK). En Economía y Empresas. Diario On Line El Libre Pensador. 12/11/2010. Accedido el 30 de enero de 2011 En:

http://www.ellibrepensador.com/2010/11/12/s indrome-de-Dunning Kruger (SDK)/.

Chiavenato, Idalberto. Administración: Teoría, procesos y práctica (3 ed.). Bogotá: McGraw Hill Interamericana. Chiavenato, I. (2006). Introducción a la Teoría General de la Administración. 7ma Edición: McGraw-Hill Interamericana.

Escoto I. (2009) ¿Conoce usted el síndrome de Dunning Kruger (SDK)? En las organizaciones el poder se obtiene en la medida en que se asciende de cargo. Diario La Prensa de Honduras. En http://www.laprensa.hn/Suplementos/Edicion es/2009/07/13/Noticias/Conoce-ustedelsindrome-de-Dunning Kruger (SDK).

Félix, S. (2007). www.arearh.com. Recuperado el 1 de junio de 2015, de www.arearh.com:http://www.arearh.com/rrhh /sindrome_Dunning Kruger (SDK).htm. 
Flores-Sandí G. (2005). Alternativa terapéutica administrativa para el "homicidio moral". Med leg Costa Rica 2005; 22: 75-80. Flores-Sandi, G. (2006). Presentismo: potencialidad en accidentes de salud. Acta méd costarric 2006; 48: 30-34

Flores-Sandí, G. (2011). Síndrome de Dunning Kruger (SDK). Acta Médica Costarricense, 53(3), 121-128.

Gray, Jeffrey A. (1971) La psicología del miedo, edic. Guadarrama. Madrid. (pp. 9-21). Guimarães LAM, Rimoli AO. (2006). "Mobbing" (Assédio Psicológico) no Trabalho: Uma Síndrome Psicossocial Multidimensional. Psic Teor e Pesq Brasilia 2006; 22: 183-192.

Jena AB, Baldwin DC Jr, Daugherty SR, Meltzer DO, Arora VM. (2010). Presenteeism Among Resident Physicians. JAMA 2010; 304: 11661168.

Martínez De Ita, María Eugenia. (2007) El concepto de productividad en el análisis Económico. Red de Estudios de la Economía Mundial. México. Disponible: http://www.redem.buap.mx/acrobat/eugenia1. pdf.

Naranjo M. (2011). ¿Cómo prevenir el síndrome de Dunning Kruger (SDK)? Semanario ForoRH. 16 de setiembre de 2010. (http://www.ForoRH.com). Accedido: 22 de febrero de 2011.2 En: http://www.rrhhmagazine.com/articulos.asp?i $\mathrm{d}=679$. Recuperado el 22 de febrero de 2011.

Nelson, D. \&. (2013). CORG, Comportamiento Organizacional (Tercera ed.). Cengage Learning, Inc. Pérez Díez de los Ríos José Luis, (1993). Modelos probabilísticos y tablas estadísticas. Mergablum. Edición y Comunicación, S.L. Rojas R.(2007). Enfoque del Presentismo en empresas de Salud. Cienc Trab 2007; 9: 64-68.

Socorro, F. (2003). El Síndrome de Dunning Kruger (SDK): el temor a ser desplazados. Extraído de: http://www.gestiopolis.com/canales/derrhh/atic ulos/51/Dunning Kruger (SDK).htm.
Socorro F. (2011). Gerentes incapazes: Reconhece algum em sua empresa? Jornal Dicas daSemana. Accedido el 28 de febrero de 2011. En http:// www.sdr.com.br/professores/FelixS/Gerentes _ineptos.htm.

Triola, M. (2013). Estadística. México: Pearson.

Pavón Y. (2010). La maldición de Dunning Kruger (SDK) - la nueva enfermedad organizacional. Degerencia.com Newsletter Mayo 2010. Accedido el 30 de enero de 2011 En:

http://www.creditperformancenews.com/es/re porter/2010- 05-00/la-maldicion-de-Dunning Kruger (SDK)-la-nueva-enfermedadorganizacional/.

Peña-Nina D. (2010). Enfermedades del liderazgo. 6 de diciembre de 2010. almomento.net. Accedido 15 de marzo de 2011. En: http://www. almomento.net/news/135/ARTICLE/75431/2 010-12-06.html.

Zapata \& Rodríguez (2008) Libro Cultura Organizacional (1) (1) - Free ebook download as PDF File (.pdf), Text File (.txt) or read book online for free. 